(C) 2014 IEEE. Personal use of this material is permitted. Permission from IEEE must be obtained for all other uses, in any current or future media, including reprinting/republishing this material for advertising or promotional purposes, creating new collective works, for resale or redistribution to servers or lists, or reuse of any copyrighted component of this work in other works 


\title{
Decentralized Control for Urban Drainage Systems Via Population Dynamics: Bogotá Case Study
}

\author{
J. Barreiro-Gomez, G. Obando, G. Riaño-Briceño, N. Quijano, and C. Ocampo-Martinez
}

\begin{abstract}
Control of Urban Drainage Systems (UDS) is studied for cases in which the distribution of run-off through the channels of a system is inefficient, i.e. when the capacity of some structures is not used optimally. In this paper, a decentralized population-dynamics-based control for UDS is presented, particularly using the replicator and projection dynamics. For the design, a methodology to make a partitioning of the system is introduced, and the design of a population-dynamics-based control per each partition is proposed. Moreover, a stability analysis of the closed-loop system is made by using passivity theory. Finally, simulation results show the proposed approach performance in a segment of the Bogotá stormwater UDS case study.
\end{abstract}

\section{INTRODUCTION}

Overflooding events in Urban Drainage Systems (UDS) occur frequently, even when the intensity of the precipitations that produce them are lower than the precipitation data that has been used to design the system [13]. Some of the main reasons of this phenomenon in urban areas are: the rapid urbanization, infrastructure complexity, and/or climate changes [13], [3]. For instance, because of the uncertainty related to the climate changes at the design stage, the expected probability of failure for hydraulic structures might increase by a factor of around 30 times [2]. For this reason, the concern of looking for new design techniques and how to address this problem with new strategies has become an important research topic in the field of hydraulic engineering. Most of the proposed solutions to solve the overflowing problem are related to procedures at the design stage [1]. However, this approach might be inefficient for already existing systems, due to the fact that this approach suggests the need of large expansions over the system. On the other hand, the active control systems approach suggests to solve the problem of overflowing by applying an optimal management of the flows throughout the pre-established system. In a centralized approach, it is costly and challenging to guarantee the availability of information in a central manner, which

J. Barreiro-Gómez and C. Ocampo-Martínez are with the Automatic Control Department, Universitat Politècnica de Catalunya, Institut de Robòtica i Informàtica Industrial (CSIC-UPC), Llorens i Artigas, 4-6, 08028 Barcelona, Spain. \{jbarreiro, cocampo\}@iri.upc.edu

J. Barreiro-Gómez, G. Obando, G. Riaño-Briceño, and N. Quijano are with Departamento de Ingeniería Eléctrica y Electrónica. G. RiañoBriceño is also with Departamento de Ingeniería Civil, Universidad de los Andes, Carrera $1^{\mathrm{A}}$ No 18A-10, Colombia \{j.barreiro135, ge-oband, ga.riano949, nquijano\}@uniandes.edu.co.

COLCIENCIAS supports J. Barreiro-Gómez and G. Obando. Agència de Gestió d'Ajust Universitaris i de Recerca AGAUR supports J. BarreiroGómez. This work has been partially supported by the projects "Drenaje urbano y cambio climático: Hacia los sistemas de alcantarillados del futuro. Fase II. COLCIENCIAS 633/2013”, ECOCIS (Ref. DPI2013-48243-C2-1R), and EFFINET (Ref. FP7-ICT-2011-8-31855). implies also a large communication network. Furthermore, it is challenging to design decentralized controllers based on partitioning due to the dynamical coupling in states that exists throughout the system. For instance, a general methodology to make a partitioning of a system in an optimal way is presented in [7]. The control approach has been widely studied by using different strategies. In [6], model predictive control is used to avoid overflows in the UDS. This control strategy is one of the most used in the literature to design the UDS control, and requires a model of the system that suitably represents its behavior. In contrast, other hydraulic problems have been solved by using other control techniques. In particular, in [8] the water distribution problem with a unique source and different receptor reservoirs has been studied with a population dynamics approach. As an advantage, the latter approach does not require of a model of the system.

The main contribution of this paper is the design of a decentralized population-dynamics-based control for UDS, that enables a better use of the existing system infrastructure. In the design process, it is proposed to make a partitioning of the UDS by using a criterion that only depends on the topology of the system, different from the one proposed in [7]. The UDS is divided into different sub-systems composed by a group of reservoirs whose outflows converge to a same receptor structure. Then, a population-dynamicsbased control is designed per each partition. The proposed population-dynamics-based controllers are designed for a topology different from the one treated in [8], where partitions are not considered, and where flows do not converge to a reservoir. Then, each local controller is in charge of making an allocation of the available volume capacities, and the different local controllers do not communicate with each other leading to a decentralized controller. Moreover, the stability of the whole closed-loop system is analyzed and proved via passivity theory, associated to the UDS and the population dynamics.

The remainder of this paper is organized as follows. Section II presents the UDS and a control-oriented model. Section III introduces the proposed partitioning methodology, and the population-dynamics-based controller. Then, stability analysis for each local closed-loop control system is presented by using passivity theory in Section IV. Section V shows the description of the Bogotá case study. In Section VI, results are shown and a discussion is made. Finally, concluding remarks and future work are presented in Section VII. 
Notation: Calligraphy letters are used to denote sets, e.g., $\mathcal{S}$. Column vectors are denoted with bold font, e.g., $\mathbf{x}$. The column vector with $n$ unitary entries is denoted by $\mathbb{1}_{n}$. The operator $\operatorname{diag}(\mathbf{x})$ is the diagonal matrix of the vector $\mathbf{x}$. Finally, $\mathbb{R}_{\geq 0}$ denotes the set of all the non-negative real number and $\mathbb{R}_{>0}$ denotes the set of all positive real numbers.

\section{URBAN DRAINAGE SYSTEMS}

In general, UDS are composed by an arrangement of channels, which are connected by inspection and collection chambers. The channel arrangement is regulated by using measuring devices and control structures to maintain specific operation conditions (e.g., velocity, Froude number, filling ratio [6]). Normally, the most suitable UDS topology is given by a tree, i.e., all channels in the UDS converge to a common drain point. In order to evaluate the performance of the system under unsteady flow conditions, two different kind of techniques can be used regarding the flow behavior representation along the channels: $i$ ) the hydraulic modeling, and $i$ ) the hydrological modeling.

The hydraulic modeling approach is mainly based on physics. This model considers the effects of the inertial and the gravitational forces over the flow. The one-dimentional Saint-Venant equations are used to model this approach subject to constraints of mass balance and momentum conservation [12]. On the other hand, the hydrological approach is based on the mass conservation principle, and it can be considered as a simplification of the hydraulic model. Thus, for the UDS modeling, the hydrological approach is useful as a qualitative solution. As an advantage, the hydrological model has a lower computational burden in comparison with the computational burden to compute a hydraulic model. For this reason, the hydrological techniques are mainly used for control-oriented models.

The purpose of the decentralized controller proposed in this paper is to allocate efficiently the fluid along the UDS channels. Then, a hydrological technique is appropriate to characterize the behavior of the system under rain (disturbance) scenarios. In order to characterize the UDS dynamics, the Muskingum model is used. Furthermore, passivity of this hydrological model is studied in Section IV.

\section{Muskingum Model}

The Muskingum model for the $i^{t h}$ reservoir is given by the differential equation $\dot{v}_{i}=I_{i}-O_{i}$, and the relation between its inflows and outflows $v_{i}=\gamma_{i}\left(I_{i} l_{i}+O_{i}\left(1-l_{i}\right)\right)$ [12], where $v_{i}$ is the volume of the reservoir, $\gamma_{i}$ is a parameter for the model calibration, $l_{i}$ is the reservoir length, and $I_{i}$ and $O_{i}$ are the inflows and outflows, respectively. Then, by expressing the outflows as function of $v_{i}, I_{i}, l_{i}$, and $\gamma_{i}$, it is obtained that $\dot{v}_{i}=\left(1-l_{i}\right)^{-1}\left(I_{i}-v_{i} / \gamma_{i}\right)$, then

$$
\dot{v}_{i}=q_{i n, i}-K_{i} x_{i} v_{i}
$$

where $q_{i n, i}=I_{i} /\left(1-l_{i}\right)$ is the inflow, $q_{o u t, i}=K_{i} x_{i} v_{i}$ is the outflow, and $K_{i} x_{i}=1 /\left(\gamma_{i}-\gamma_{i} l_{i}\right)$. Furthermore, $x_{i} \in[0,1]$ determines the control action over the output gate, which is related to its percentage of opening, i.e,, zero is completely closed, and one is completely opened. Moreover $K_{i}>0$ scales the outflow, and it can be seen as the discharge coefficient of the reservoir.

\section{Control Via Population Dynamics}

The proposed control approach consists in dividing the UDS into $m$ different sub-systems with the same local topology. Each local topology has different source reservoirs and one receptor. Moreover, a population-dynamics-based controller is designed for each sub-system. In fact, the population dynamics approach has been already used in hydraulic systems [8]. However, the authors in [8] deal with a different topology (i.e., one source reservoir and different receptor reservoirs), and a different control goal without performing the partitioning of the system. The approach proposed in this paper considers a decentralized control scheme for the UDS that is composed by $m$ local controllers that do not communicate with each other and operate independently in parallel. This section introduces the partitioning criterion of the UDS into sub-systems. Furthermore, the population dynamics used in the control design are presented.

\section{A. System Partitioning}

Consider a simple UDS sub-system composed by $n$ source reservoirs, and only one receptor reservoir as shown in the partition 1 of Figure 1. This topology is known as the case of flow convergence ${ }^{1}$. In this case, let $\mathcal{S}=\{1, \ldots, n\}$ be the set of source reservoirs. The maximum volume of the $i^{t h}$ reservoir is denoted by $\bar{v}_{i}$. The control objective consists in avoiding overflows throughout the system, i.e., to avoid that the current volume of the reservoir $v_{i}$ never surpasses its maximum volume capability $\bar{v}_{i}$, for all $i \in \mathcal{S}$. To achieve this objective, it is proposed to do an allocation of the available capability of the $n$ reservoirs, i.e., to distribute the current available volume given by $\bar{v}_{i}-v_{i}$ in an optimal way by controlling the outflows $q_{\text {out }, i}$, for all $i \in \mathcal{S}$.

Each sub-system must correspond to a case of flow convergence. In order to clarify the partitioning process in a typical tree topology of a UDS, an arbitrary tree UDS is presented in Figure 1. At this general example, there are two stages and three partitions. Notice that the subsystem corresponding to each partition fits the case of flow convergence. In this process, it is possible to find a partitioning in which a reservoir is a source and also a receptor for different partitions in the UDS. For instance, in the partitioning presented in Figure 1, the gray reservoirs are receptors for the partitions 1 and 2 , and source reservoirs for the partition 3 .

\section{B. Population Dynamics Approach}

Consider a population composed by a large and finite number of agents. Assume that the total amount of agents represents the total outflow from all the source reservoirs to the receptor reservoir, which is given by a complete opening of the gates in the sub-system outflows. Suppose

\footnotetext{
${ }^{1}$ Due to the fact that there are several outflows converging to a unique receptor reservoir.
} 


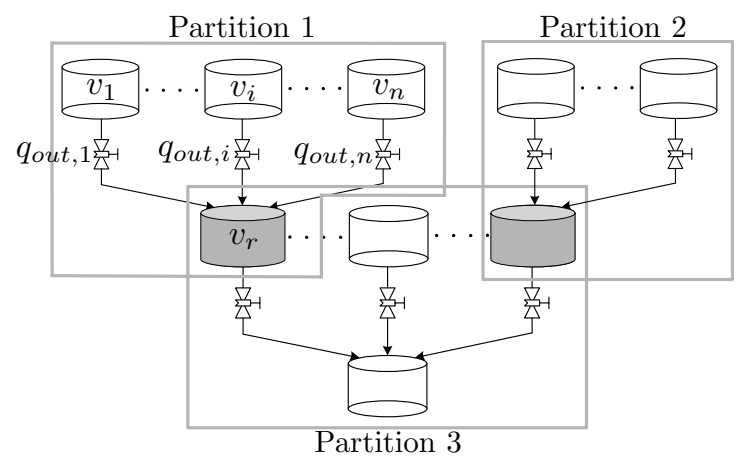

Fig. 1. Partitions over a tree topology. Each partition corresponds to a flow-convergence topology, with $n$ source reservoirs and one receptor reservoir. Some reservoirs are source and receptor in different partitions (gray reservoirs).

that agents can select an outflow corresponding to a source reservoir from the set of $n$ reservoirs $\mathcal{S}$. In the population context, $\mathcal{S}$ represents the set of strategies. The scalar value $x_{i} \in \mathbb{R}_{\geq 0}$ represents the proportion of agents selecting the reservoir $i \in \mathcal{S}$, i.e., the opening proportion of the $i^{t h}$ outflow gate is $x_{i}$. The population state $\mathbf{x} \in \mathbb{R}_{\geq 0}^{n}$ represents all the proportions assigned to the reservoirs, i.e., $\mathbf{x}=\left[x_{1}, \ldots, x_{n}\right]^{\top}$. Since $\mathbf{x}$ represents proportions, then the set of possible population states is given by the simplex $\Delta=\left\{\mathbf{x} \in \mathbb{R}_{\geq 0}^{n}: \mathbf{x}^{\top} \mathbb{1}_{n}=1\right\}$. Agents in the population have incentives to select the reservoir outflows (e.g., in a general control system, the error is an incentive for the controller to apply more energy to the system and then correct the states to achieve the desired values). The incentives associated to a rewarding that the proportion of agents $x_{i}$ receives for selecting the reservoir $i \in \mathcal{S}$ are given by a fitness function $f_{i}(\mathbf{x})$, whose mapping is $f_{i}: \Delta \mapsto \mathbb{R}$. Moreover, the vector of all the fitness functions is denoted by $\mathbf{F}=\left[\begin{array}{lll}f_{1} & \cdots & f_{n}\end{array}\right]^{\top}$ with mapping $\mathbf{F}: \Delta \mapsto \mathbb{R}^{n}$. The following fitness function is proposed to control the case of flow convergence topology:

$$
f_{i}=-\left(\frac{1}{A \frac{v_{i}}{\bar{v}_{i}}+\epsilon}\right) x_{i},
$$

where $A$ determines the slope rate of the fitness function, and $\epsilon \in \mathbb{R}_{>0}$ is a small factor that avoids an indetermination of $f_{i}$ when $v_{i}=0$. Moreover, the proposed fitness function for the strategy $i \in \mathcal{S}$ only depends on the volume $v_{i}$ and the proportion of agents $x_{i}$, making it suitable for applying in cases where only local information is available. The solution of the population game is a Nash equilibrium denoted by $\mathbf{x}^{*} \in \Delta$ in which no agent has incentives to switch from one strategy to another [9]. Indeed, the solution is characterized by the condition $f_{i}=f_{j}$, for all $i, j \in \mathcal{S}$.

Each partition of the UDS is controlled by a population dynamics system as presented in Figure 2. All the gates, defining the outflow of the source reservoir in a partition, are established by the vector $\mathbf{x} \in \mathbb{R}^{n}$. These opening grades of the output gates affect the behavior of the reservoir volumes, i.e., $\mathbf{v}=\left[v_{1}, \ldots, v_{n}\right]^{\top}$. Hence, the variation of the reservoir volumes modifies the fitness function (2), affecting the control actions over the output gates $\mathbf{x} \in \mathbb{R}^{n}$.

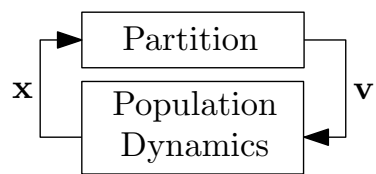

Fig. 2. Closed-loop control for a partition of the UDS.

For the population dynamics design, it is proposed to work with two kinds of population dynamics: the replicator dynamics introduced by Taylor and Jonker in [11], given by

$$
\dot{\mathbf{x}}=\operatorname{diag}(\mathbf{x})\left(\mathbf{F}-\mathbb{1}_{n} \mathbf{x}^{\top} \mathbf{F}\right)
$$

and the projection dynamics introduced by Nagurney and Zhang in [4], given by

$$
\dot{\mathbf{x}}=\mathbf{F}-\frac{1}{n} \mathbb{1}_{n} \mathbf{F}^{\top} \mathbb{1}_{n}
$$

These dynamics are of interest in this work since they share gradient properties [10], and because of their passivity properties, which are studied in Section IV.

\section{Stability Analysis}

This section studies the stability properties of the closedloop system controlled via replicator/projection dynamics.

\section{A. Equilibrium Point}

First, the equilibrium point is characterized.

Proposition 1: Let $f^{*} \in \mathbb{R}$ be a constant. If $\mathrm{x}^{*} \in$ int $\Delta$, where int $\Delta=\left\{\mathbf{x}^{*} \in \mathbb{R}_{>0}^{n}: \mathbb{1}_{n}^{\top} \mathbf{x}^{*}=1\right\}$, then the equilibrium point $\left(\mathbf{v}^{*}, \mathbf{x}^{*}\right)$ of the closed-loop system controlled via population dynamics (i.e., the closed-loop system given by (1) and (3) or (4)) satisfies the following property:

$$
f_{i}\left(v_{i}^{*}, x_{i}^{*}\right)=f^{*}, \text { for all } i=1, \ldots, n,
$$

where $v_{i}^{*}$ and $x_{i}^{*}$ denotes the $i^{t h}$ element of the vectors $\mathbf{v}^{*}$ and $\mathrm{x}^{*}$, respectively.

Proof: Consider the replicator dynamics (3). Since $\mathbf{x}^{*} \in$ int $\Delta$ by assumption, $\dot{\mathbf{x}}=0$ if and only if $\mathbf{F}^{*}=$ $\mathbb{1}_{n} \mathbf{x}^{* \top} \mathbf{F}^{*}$, where $\mathbf{F}^{*}$ denotes the vector of fitness functions in steady state. Taking $\mathbf{x}^{* \top} \mathbf{F}=f^{*}$, it is concluded that $f_{i}\left(v_{i}^{*}, x_{i}^{*}\right)=f^{*}$, for all $i=1, \ldots, n$.

Furthermore, consider the projection dynamics (4). $\dot{\mathbf{x}}=0$ if and only if $n \mathbf{F}^{*}=\mathbb{1}_{n}^{\top} \mathbf{F}^{*} \mathbb{1}_{n}$. Taking $\mathbb{1}_{n}^{\top} \mathbf{F}^{*}=n f^{*}$, it is concluded that $f_{i}\left(v_{i}^{*}, x_{i}^{*}\right)=f^{*}$, for all $i=1, \ldots, n$.

It is worth noting that the fitness functions associated with the reservoirs are equalized at equilibrium, i.e., $\mathrm{x}^{*}$ is a Nash equilibrium of the underlying population game. The fact that the fitness functions achieve a same value means that there is a well-balanced assignment of the source reservoir outflows with respect to their current volumes, i.e., there is an equitable distribution of the available volume capacities in the reservoirs. This fact is the core of the proposed control strategy designed to avoid overflows throughout the UDS. 


\section{B. Stability}

Now, passivity theory is used in order to study the stability properties of the equilibrium point characterized before.

Notice that, the UDS dynamics can be written in error coordinates as follows:

$$
\dot{e}_{v_{i}}=-k_{i} e_{v_{i}}\left(x_{i}-x_{i}^{*}\right)-k_{i} x_{i}^{*} e_{v_{i}}-k_{i} v_{i}^{*} e_{x_{i}}, i \in \mathcal{S},
$$

where $e_{v_{i}}=v_{i}-v_{i}^{*}$, and $\left(v_{1}^{*}, \ldots, v_{n}^{*}, x_{1}^{*}, \ldots, x_{n}^{*}\right)$ is the equilibrium point given in Proposition 1. Moreover, notice that $\dot{e}_{v_{i}}=\dot{v}_{i}$ since $\dot{v}_{i}^{*}=0$. An important property of this system is provided in the following lemma.

Lemma 1: Assume that $x_{i}(t) \geq 0$, for all $i=1, \ldots, n$; and $v_{i}(t)>0$, for all $i=1, \ldots, n$, and for all $t \geq 0$. The system given in (6) is passive if its input is defined as $\mathbf{x}-\mathbf{x}^{*}=\left[x_{1}-x_{1}^{*}, \ldots, x_{n}-x_{n}^{*}\right]^{\top}$ and its output as $-\mathbf{g}_{\mathbf{e}_{\mathbf{v}}}=-\left[g_{1}\left(e_{v_{1}}\right), \ldots, g_{n}\left(e_{v_{n}}\right)\right]^{\top}$, where $g_{i}(\cdot)$ is null in zero and strictly increasing continuous function for all $i=1, \ldots, n$. Furthermore, the corresponding storage function is positive definite.

Proof: The proof uses the following storage function (adapted from [5]):

$$
V_{1}\left(\mathbf{e}_{\mathbf{v}}\right)=\sum_{i=1}^{n} \frac{1}{k_{i} v_{i}^{*}} \int_{0}^{e_{v_{i}}} g_{i}(\delta) d \delta .
$$

$V_{1}\left(\mathbf{e}_{\mathbf{v}}\right)$ is positive definite since: $\left.i\right)$ it is strictly convex because the derivative of each term in (7) is strictly increasing by assumption; and ii) its minimum is at $\mathbf{e}_{\mathbf{v}}=0$, i.e., $\left.\nabla V\right|_{\mathbf{e}_{\mathbf{v}}=0}=0$.

The derivative of $V_{1}\left(\mathbf{e}_{\mathbf{v}}\right)$ along the trajectories of the system stated in (6) is given by $\dot{V}_{1}\left(\mathbf{e}_{\mathbf{v}}\right)=\sum_{i=1}^{n} \frac{e_{v_{i}}}{k_{i} v_{i}^{*}} \dot{e}_{v_{i}}$, then $\dot{V}_{1}\left(\mathbf{e}_{\mathbf{v}}\right)=-\sum_{i=1}^{n}\left(x_{i}-x_{i}^{*}\right) g_{i}\left(e_{v_{i}}\right)-\sum_{i=1}^{n} \frac{x_{i}}{v_{i}^{*}} e_{v_{i}} g_{i}\left(e_{v_{i}}\right)$, and

$$
\dot{V}_{1}\left(\mathbf{e}_{\mathbf{v}}\right)=-\mathbf{g}_{\mathbf{e}_{\mathbf{v}}}{ }^{\top}\left(\mathbf{x}-\mathbf{x}^{*}\right)-\sum_{i=1}^{n} \frac{x_{i}}{v_{i}^{*}} e_{v_{i}} g_{i}\left(e_{v_{i}}\right),
$$

where $\sum_{i=1}^{n} \frac{x_{i}}{v_{i}^{*}} e_{v_{i}} g_{i}\left(e_{v_{i}}\right) \geq 0$ since it is assumed that $v_{i}^{*}>$ 0 and $x_{i} \geq 0$. Thus, $\dot{V}_{1}\left(\mathbf{e}_{\mathbf{v}}\right) \leq-\mathbf{g}_{\mathbf{e}_{\mathbf{v}}}{ }^{\top}\left(\mathbf{x}-\mathbf{x}^{*}\right)$. Therefore, the system is passive.

Additionally, the controllers based on population dynamics described in (3) and (4) exhibit similar properties. These properties are summarized in Lemmas 2 and 3.

Lemma 2: The replicator dynamics system (3) is lossless if its input is defined as $\mathbf{F}-\mathbb{1}_{n} f^{*}=\left[f_{1}-f^{*}, \ldots, f_{n}-\right.$ $\left.f^{*}\right]^{\top}$ and its output as $\mathbf{x}-\mathbf{x}^{*}=\left[x_{1}-x_{1}^{*}, \ldots, x_{n}-x_{n}^{*}\right]^{\top}$. Furthermore, the storage function is positive definite.

Proof: First, notice that adding the same constant to all fitness function does not affect the behavior of replicator equation in the simplex $\Delta$. Therefore, the system in (3) can be rewritten as follows:

$$
\dot{x}_{i}=x_{i}\left(f_{i}-f^{*}-\mathbf{x}^{\top}\left(\mathbf{F}-\mathbb{1}_{n} f^{*}\right)\right), i \in \mathcal{S} .
$$

Now, let us prove that this system is lossless from the input $\mathbf{F}-f^{*}$ to the output $\mathbf{x}-\mathbf{x}^{*}$. In order to do this, we take the following positive definite storage function is taken:

$$
V_{2}(\mathbf{x})=-\sum_{i=1}^{n} x_{i}^{*} \ln \left(\frac{x_{i}}{x_{i}^{*}}\right) .
$$

Notice that $V_{2}(\mathbf{x})=0$ for $\mathbf{x}=\mathbf{x}^{*}$, and $V_{2}(\mathbf{x})>0$ for $\mathbf{x} \neq \mathbf{x}^{*}$. The derivative of $V_{2}(\mathbf{x})$ along the trajectories of (8) is given by $\dot{V}_{2}(\mathbf{x})=-\sum_{i=1}^{n} \frac{x_{i}^{*}}{x_{i}} \dot{x}_{i}$, then $\dot{V}_{2}(\mathbf{x})=-\sum_{i=1}^{n} x_{i}^{*}\left(f_{i}-f^{*}-\mathbf{x}^{\top}\left(\mathbf{F}-\mathbb{1}_{n} f^{*}\right)\right)$. This leads to obtain $V_{2}(\mathbf{x})=\sum_{i=1}^{n}\left(x_{i}-x_{i}^{*}\right)\left(f_{i}-f^{*}\right)$. Finally

$$
V_{2}(\mathbf{x})=\left(\mathbf{F}-\mathbb{1}_{n} f^{*}\right)^{\top}\left(\mathbf{x}-\mathbf{x}^{*}\right) .
$$

Thus, the system is lossless.

Lemma 3: The projection dynamics system (4) is lossless if its input is defined as $\mathbf{F}-\mathbb{1}_{n} f^{*}=\left[f_{1}-f^{*}, \ldots, f_{n}-\right.$ $\left.f^{*}\right]^{\top}$ and its output as $\mathbf{x}-\mathbf{x}^{*}=\left[x_{1}-x_{1}^{*}, \ldots, x_{n}-x_{n}^{*}\right]^{\top}$. Furthermore, the storage function is positive definite.

Proof: First, notice that adding the same constant to all fitness function does not affect the behavior of projection dynamics. Therefore, the system in (4) can be rewritten as follows:

$$
\dot{x}_{i}=f_{i}-f^{*}-\frac{1}{n} \mathbb{1}_{n}\left(\mathbf{F}-\mathbb{1}_{n} f^{*}\right)^{\top} \mathbb{1}_{n},
$$

for all $i \in \mathcal{S}$. Now, let us prove that this system is lossless from the input $\mathbf{F}-f^{*}$ to the output $\mathbf{x}-\mathbf{x}^{*}$. In order to do this, take the following positive definite storage function [9]:

$$
V_{3}(\mathbf{x})=\frac{1}{2} \sum_{i=1}^{n}\left(x_{i}-x_{i}^{*}\right)^{2} .
$$

Notice that $V_{3}(\mathbf{x})=0$ for $\mathbf{x}=\mathbf{x}^{*}$, and $V_{3}(\mathbf{x})>0$ for $\mathbf{x} \neq \mathbf{x}^{*}$. The derivative of $V_{3}(\mathbf{x})$ along the trajectories of (10) is given by $\dot{V}_{3}(\mathbf{x})=\sum_{i=1}^{n}\left(x_{i}-x_{i}^{*}\right) \dot{x}_{i}$. Then, it follows that

$$
\dot{V}_{3}(\mathbf{x})=-\frac{1}{n}\left(\mathbf{F}-\mathbb{1}_{n} f^{*}\right)^{\top} \mathbb{1}_{n} \sum_{i=1}^{n}\left(x_{i}-x_{i}^{*}\right)+\Psi(\mathbf{x}),
$$

where $\Psi(\mathbf{x})=\sum_{i=1}^{n}\left(x_{i}-x_{i}^{*}\right)\left(f_{i}-f^{*}\right)$, then

$$
\dot{V}_{3}(\mathbf{x})=-\frac{1}{n}\left(\mathbf{F}-\mathbb{1}_{n} f^{*}\right)^{\top} \mathbb{1}_{n}\left(\sum_{i=1}^{n} x_{i}-\sum_{i=1}^{n} x_{i}^{*}\right)+\Psi(\mathbf{x}) .
$$

Due to the fact that $\sum_{i=1}^{n} x_{i}=\sum_{i=1}^{n} x_{i}^{*}$, then $\dot{V}_{3}(\mathbf{x})=\sum_{i=1}^{n}\left(x_{i}-x_{i}^{*}\right)\left(f_{i}-f^{*}\right)$. Finally

$$
\dot{V}_{3}(\mathbf{x})=\left(\mathbf{F}-\mathbb{1}_{n} f^{*}\right)^{\top}\left(\mathbf{x}-\mathbf{x}^{*}\right) .
$$

Hence, the system is lossless.

The following results regarding the stability properties of the closed-loop system are based on passivity of the UDS and controllers.

Theorem 1: The equilibrium point $\left(\mathbf{v}^{*}, \mathbf{x}^{*}\right)$, characterized in Proposition 1, of the closed-loop system controlled via replicator dynamics is stable.

Proof: It is used $V=V_{1}+V_{2}$ as a Lyapunov function candidate, where $V_{1}$ and $V_{2}$ are given in (7), (9), respectively. According to Lemma 1 and Lemma 2, it is known that the derivative of $V$ along the trajectories of the closed-loop system is given by $\dot{V} \leq-\mathbf{g}_{\mathbf{e}_{\mathbf{v}}}{ }^{\top}\left(\mathbf{x}-\mathbf{x}^{*}\right)+\left(\mathbf{F}-\mathbb{1}_{n} f^{*}\right)^{\top}(\mathbf{x}-$ $\mathbf{x}^{*}$ ). Taking $\mathbf{g}_{\mathbf{e}_{\mathbf{v}}}=\mathbf{F}-\mathbb{1}_{n} f^{*}$ (notice that $i^{t h}$ element of the vector $\mathbf{F}-\mathbb{1}_{n} f^{*}$ satisfies the conditions in Lemma 1, i.e., $f_{i}-f^{*}$ is continuously increasing and zero in $v_{i}=v_{i}^{*}$ ), obtaining $\dot{V} \leq 0$. Therefore, the system is stable. 

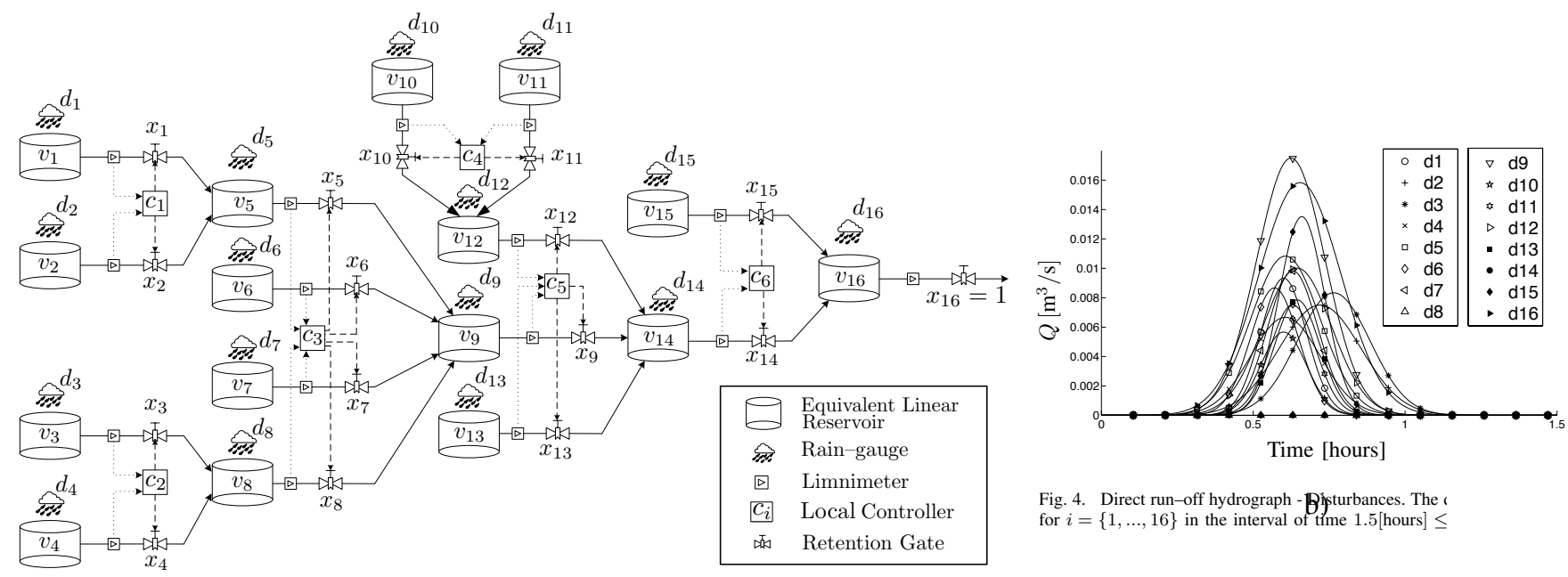

b)

a)

Fig. 3. a) Equivalent system of a portion of the Bogotá, Chicó UDS. b) Direct run-off hydrograph - Disturbances. The disturbances $d_{i}=0$ for $i=\{1, \ldots, 16\}$ in the interval of time 1.5 hours $\leq t \leq 5.25$ hours. Furthermore, control action $x_{16}=1$ in order to evacuate waste water from the network after the flow management.

Theorem 2: The equilibrium point $\left(\mathbf{v}^{*}, \mathbf{x}^{*}\right)$, characterized in Proposition 1, of the closed-loop system controlled via projection dynamics is stable.

Proof: The procedure follows the proof of Theorem 1.

\section{CAse Study}

It has been shown in Section IV that the proposed closedloop control system for each partition is stable. Then, the proposed controller is applied for a case study to illustrate the usefulness of the model-free decentralized populationdynamics-based controller for UDS. The proposed case study consists of a part of the Bogotá (Colombia) stormwater UDS shown in Figure 3a). The UDS is composed by 16 sub-catchments that drain to 16 collection chambers. The groups of catchments are approximated to reservoirs as in [6], and the system dynamics are modeled with the Muskingum reservoir model presented in Section II. Each equivalent reservoir represents a source or receptor reservoir of a partition of the system as it is stated in Section IIIA. The system is modeled for a typical rain scenario, in which flooding occurs. The direct run-off hydrographs that represent the system disturbances are presented in Figure 3b). The maximum run-off peak is approximately $18 \mathrm{l} / \mathrm{s}$.

Additionally, the maximum volumes $\bar{v}_{i}$, and the $K_{i}$ constants for each reservoir in the UDS are presented in Table I. The $K_{i}$ constants have been found by using EPA-SWMM, which is a computer program specialized in hydraulic modeling. The program has been used to retrieve flow and volume information, given the geometric features of the network. Since $q_{o u t, i}=K_{i} v_{i}$ when the $i^{t h}$ gate is fully opened, $K_{i}$ can be easily calculated assuming linearity. Moreover, it is assumed that there is full information about measured reservoir volumes within a partition, i.e., each local controller has full information about the source tanks within the partition. The capacity of each reservoir in the system is checked during the run-off routing process, for a 5.25 hours time window. The capacity is defined as the normalized volume of the $i^{t h}$ reservoir, i.e., $v_{\text {normalized }}=v_{i} / \bar{v}_{i}$. Then, a flooding event occurs when the volume in a reservoir exceeds its maximum volume, i.e., when $v_{\text {normalized }}>1$.

\section{TABLE I}

\begin{tabular}{c|cc||c|cc} 
Res. & $\begin{array}{c}\text { Coeffi. } \\
K_{i}\end{array}$ & $\begin{array}{c}\text { max. vol. } \\
\bar{v}_{i}\left[\mathrm{~m}^{3}\right]\end{array}$ & Res. & $\begin{array}{c}\text { Coeffi. } \\
K_{i}\end{array}$ & $\begin{array}{c}\text { max. vol. } \\
\bar{v}_{i}\left[\mathrm{~m}^{3}\right]\end{array}$ \\
\hline \hline 1 & 0.002332 & 114.50 & 9 & 0.006147 & 274.80 \\
2 & 0.003870 & 137.40 & 10 & 0.005446 & 183.20 \\
3 & 0.003170 & 114.50 & 11 & 0.020703 & 183.20 \\
4 & 0.008239 & 125.95 & 12 & 0.001693 & 274.80 \\
5 & 0.002217 & 274.80 & 13 & 0.007026 & 274.80 \\
6 & 0.008975 & 274.80 & 14 & 0.000632 & 807.22 \\
7 & 0.005185 & 274.80 & 15 & 0.006319 & 274.80 \\
8 & 0.004764 & 274.80 & 16 & 0.005782 & 274.80 \\
\hline
\end{tabular}

Res.=Reservoir, max. vol.= maximum volume

The control objective is to avoid overflows throughout the UDS. To do so, it is proposed to take advantage of all the available volume capacities in the whole network. At each partition, there is a controller managing the outflows of the source reservoirs as it is described in Section III.

\section{RESULTS AND DISCUSSION}

The system is tested for a typical rain scenario (see Figure $3 b)$ ). The results are analyzed by making a comparison between the case without control (no active elements), and the case applying the decentralized population dynamicsbased control. The results show the total flood volume and the capacity of each reservoir in the network. The biggest and smallest reservoirs have a capacity of $807.22 \mathrm{~m}^{3}$ and $114.5 \mathrm{~m}^{3}$, respectively (see Table I). Table II shows the total overflow for the case study. When there is no control, the overflow is 1165.281 and null when the proposed decentralized control strategy is applied. It can be seen that the control strategy is quite useful in the reduction of overflows for the case study. 


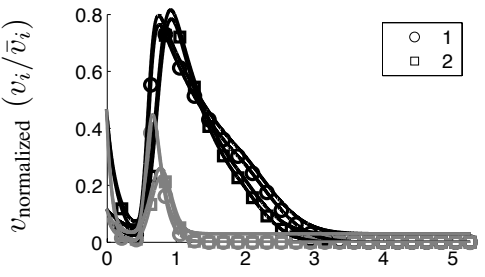

a)

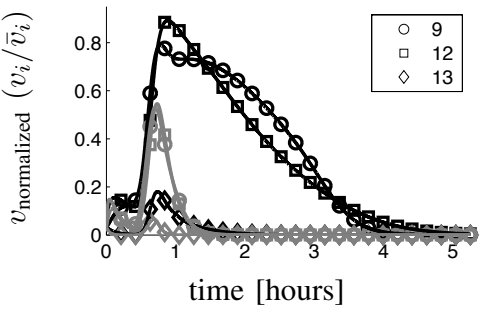

d)

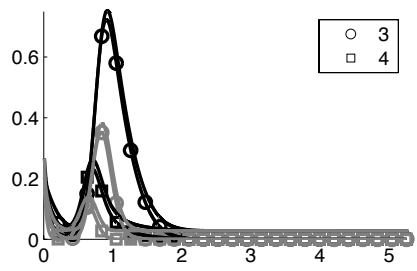

b)

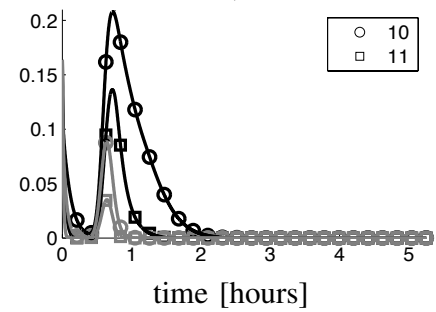

e)

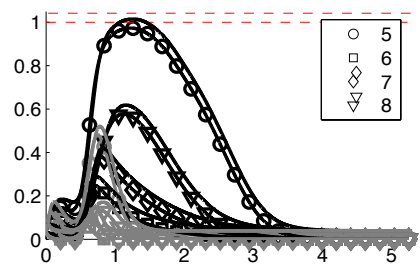

c)

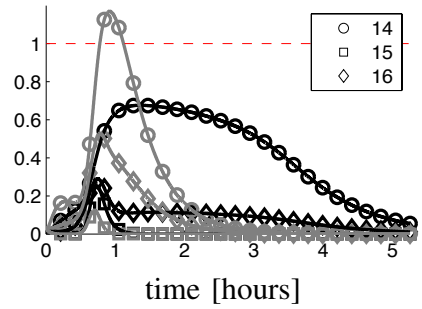

f)

Fig. 4. Evolution of the 16 normalized reservoir volumes. Gray lines correspond to a scenario without control, and black lines correspond to a scenario with the decentralized controller. Figures a), b), c), d), e), and f) show the reservoirs for the controllers $1,2,3,4,5$, and 6 , respectively. Labels refer to the different reservoirs in the UDS.

\begin{tabular}{l|c} 
Control case & Overflows 1 \\
\hline \hline Without Control & 1165.28 \\
Decentralized with Population Dynamics & 0 \\
\hline
\end{tabular}

Additionally, the evolution of the UDS capacities is presented in Figure 4. It is shown that stormwater is better distributed throughout the network for the decentralized control case. The upstream reservoirs get more filled and their capacity utilization increases to almost the double. The flow downstream is relieved and flooding is avoided. The use of the proposed decentralized controllers and the cascade topology guarantees that the capacity of the reservoirs upstream is more effectively used. Furthermore, when the system is not controlled, the time it takes to reach the steady state is close to 2.5 hours. The basic hydraulic design is oriented to evacuate the run-off as fast as possible. However, this design approach can lead to flooding events in terminal nodes as seen in Figure 4f). In the case where control is used, reaching the steady state for reservoirs takes more time compared to the uncontrolled case, and this is a consequence of the retaining property provided by the controller. The control objective is satisfied, i.e., reducing overflows by distributing better the water resource.

\section{CONCLUSIONS AND FURTHER WORK}

It has been shown that the proposed decentralized population-dynamics-based control is efficient in terms of a better distribution of wastewater throughout the UDS, avoiding overflows. The partitioning proposed methodology allows to design the decentralized control scheme by using different local controllers with a lower computational burden with respect to a centralized control scheme. Furthermore, it has been shown that the proposed closed-loop system is stable since the UDS and population-dynamics-based controllers have passivity properties. As further work, it is proposed to implement the decentralized population dynamics-based control in a larger-scale UDS problem (e.g., a bigger portion of the Bogotá UDS) by taking advantage of the passivity properties of the closed-loop system. Additionally, some of the issues related to the evacuation of the wastewater in reservoirs might be studied by adding a multi-objective controller based on population dynamics, e.g., it would be appropriate to establish an additional control objective related to evacuation rate flow throughout the network.

\section{REFERENCES}

[1] K. Arnbjerg-Nielsen, P. Willems, J. Olsson, S. Beecham, A. Pathirana, I.B. Gregersen, H. Madsen, and V. Nguyen. Impacts of climate change on rainfall extremes and urban drainage systems: a review. Water Science \& Technology, 68(1):16-28, 2013.

[2] R. Ashley, D. Balmforth, A. Saul, and J. Blanskby. Flooding in the future predicting climate change, risks and responses in urban areas. Water Science \& Technology, 52(5):265-273, 2005.

[3] K. Berggren, M. Olofsson, M. Viklander, G. Svensson, and A. Gustafsson. Hydraulic impacts on urban drainage systems due to changes in rainfall caused by climatic change. Journal of Hydrologic Engineering, 17(1):92-98, 2011.

[4] A. Nagurney and D. Zhang. Projected dynamical systems in the formulation, stability analysis, and computation of fixed demand traffic network equilibria. Transportation Science, 31:147-158, 1997.

[5] G. Obando, A. Pantoja, and N. Quijano. Building Temperature Control based on Population Dynamics. IEEE Transactions on Control Systems Technology., 22(1):404-412, 2014.

[6] C. Ocampo-Martinez. Model predictive control of wastewater systems. Springer, 2010.

[7] C. Ocampo-Martinez, S. Bovo, and V. Puig. Partitioning approach oriented to the decentralised predictive control of large-scale systems. Journal of Process Control, 21(5):775-786, 2011.

[8] E. Ramirez-Llanos and N. Quijano. A population dynamics approach for the water distribution problem. International Journal of Control, 83(9): 1947-1964, 2010.

[9] W. H. Sandholm. Population games and evolutionary dynamics. Cambridge, Mass. MIT Press, 2010.

[10] W. H. Sandholm, E. Dokumaci, and R. Lahkar. The projection dynamic and the replicator dynamic. Games and Economic Behavior, 64(2):666-683, 2008.

[11] P. D. Taylor and L. B. Jonker. Evolutionary stable strategies and game dynamics. Mathematical biosciences, 40(1):145-156, 1978.

[12] V. Te Chow. Open channel hydraulics. McGraw-Hill Book Company, Inc; New York, 1959.

[13] P. Willems, K. Arnbjerg-Nielsen, J. Olsson, and V. Nguyen. Climate change impact assessment on urban rainfall extremes and urban drainage: Methods and shortcomings. Atmospheric research, 103:106$118,2012$. 